\title{
EXTENDED DUST SHELLS AROUND CARBON STARS IN THE INFRARED AND IN OPTICAL LIGHT
}

\author{
HIDEYUKI IZUMIURA \\ Okayama Astrophysical Observatory, NAOJ \\ Kamogata, Asakuchi, Okayama 719-0232, Japan \\ L. B.F.M. WATERS AND T. DE JONG \\ Astronomical Institute "Anton Pannekoek", \\ University of Amsterdam \\ NL-1098 SJ Amsterdam, The Netherlands \\ C. LOUP \\ Institut d'Astrophysique de Paris, \\ CNRS, 75014 Paris, France \\ AND \\ O. HASHIMOTO \\ Gunma Astronomical Observatory, \\ 1-18-7 Ohotomo, Maebashi, Gunma 371, Japan
}

\begin{abstract}
We investigate the structure of extended dust shells around optical carbon stars in the far-infrared and in optical light. In the optical we have discovered that $\mathrm{R} \mathrm{Scl}$ and $\mathrm{U}$ Ant are associated with circularly extended emission, the radii of which are about $20^{\prime \prime}$ and $58^{\prime \prime}$, respectively. The emission is probably scattered light of the central star by dust grains in their circumstellar shells. In the far-infrared we have discovered a double shell structure surrounding U Ant in high resolution IRAS images, which is direct evidence of a periodic change of mass-loss on a time-scale of the order of $10^{4}$ years in the AGB evolution. Relating the two shells to two consecutive thermal pulses allows for a self-consistent determination of the interpulse period, core-mass, luminosity, and distance. Direct mapping of Y CVn at 90 and $160 \mu \mathrm{m}$ with ISOPHOT on board ISO has revealed a very extended detached dust shell around this star. The mass-loss rate is found to have decreased drastically by two orders of magnitude $1.4 \times 10^{4}$ years ago, which should be an important clue to the understanding of J-type stars.
\end{abstract}




\section{Introduction}

It is widely accepted that carbon stars are produced from low- and intermediate-mass stars by the third dredge-up at the thermally pulsing asymptotic giant branch (TP-AGB) phase (Iben \& Renzini 1983). Such TP-AGB stars undergo intense mass-loss, which gives rise to a copious circumstellar shell of gas and dust. If very extended dust shells indeed exist around carbon stars, they must be one of the best sites for the study of the actual interplay between thermal pulses and mass-loss, which is now thought to be a key process in understanding the AGB evolution. Examining the structure of such a dust shell will allow us to obtain mass-loss history on time scales of 1,000 to 10,000 years. In the following sections we describe our recent results on the extended dust shells of some carbon stars.

\section{Dust Shells of R Scl and U Ant in Optical Light}

In Figure 1 we show images of $\mathrm{R} \mathrm{Scl}$ and $\mathrm{U}$ Ant in blue light retrieved from the Digitized Sky Surveys. In the images we find that these stars are surrounded by an extended circular emission centered at the star. For R Scl the emission is smaller than a ghost pattern which is also seen for the star of similar magnitude at the right in the same panel. For U Ant the emission extends outside the ghost pattern.

The images were recorded on SERC-J (blue) plates taken with the UK Schmidt Telescope. For each star we examined images of other stars with similar brightness on both the same and different SERC-J survey plates. We did not find any similar extended emisson components. We therefore conclude that the extended components around R Scl and U Ant are true extended emission, probably the light from the central stars scattered by dust grains in their circumstellar dust shells.

The radii of the optical shells are $20^{\prime \prime}$ and $58^{\prime \prime}$ for $\mathrm{R} \mathrm{Scl}$ and U Ant, respectively, which are in close agreement with those of their $\mathrm{CO}$ gas shells of $9^{\prime \prime}+(5-10)^{\prime \prime}$ and $41^{\prime \prime}+(5-10)^{\prime \prime}$, respectively (Olofsson et al. 1996). This excellent agreement between the shell sizes in the optical and $\mathrm{CO}$ gas emission strongly supports our idea that the extended emission in the blue optical light is due to light scattered by dust grains.

It is notable, however, that the dust emission is slightly but significantly more extended than the $\mathrm{CO}$ gas emission in both stars. The outer boundary of the $\mathrm{CO}$ gas shell may be truncated by the photodissociation process by the interstellar UV radiation field. Another possibility is the gas/dust drift (Gilman 1972; Goldreich \& Scoville 1976; Kwan \& Hill 1977), which has long been suggested but never measured directly. The drift velocity can be as large as $9 \mathrm{~km} \mathrm{~s}^{-1}$ or $5 \mathrm{~km} \mathrm{~s}^{-1}$ when the momentum transfer efficiency factor is 0.05 (Sopka et al. 1985) or 0.015 (Huggins, Olofsson \& Johansson 


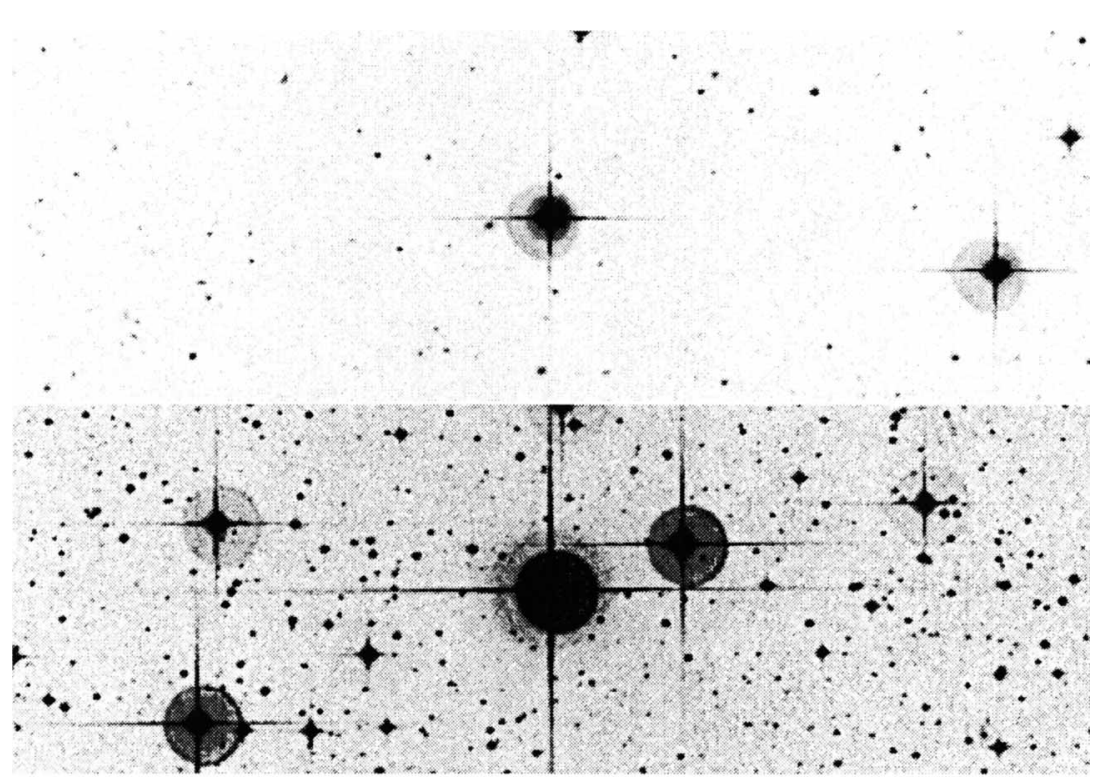

Figure 1. Top: Dust shell image of $\mathrm{R} \mathrm{Scl} \mathrm{recorded} \mathrm{on} \mathrm{a} \mathrm{blue} \mathrm{photographic} \mathrm{plate} \mathrm{retrieved}$ from the Digitized Sky Survey. Bottom: Same as the top panel, but for U Ant. The images measure $5^{\prime} .12 \times 15^{\prime} .0$ and the carbon stars are at the center.

1988), respectively, assuming a mass-loss rate of $5 \times 10^{-6} \mathrm{M}_{\odot} \mathrm{yr}^{-1}$, a gas expansion velocity of $21 \mathrm{~km} \mathrm{~s}^{-1}$, and a luminosity of $1.7 \times 10^{4} \mathrm{~L}_{\odot}$ (Izumiura et al. 1997). Hence the size difference could be due to the gas/dust drift.

\section{HIRAS Images of the Dust Shell of U Ant}

High resolution IRAS (HIRAS) images in the far-infrared are now available with the use of Pyramid Maximum Entropy image reconstruction techniques (Bontekoe et al. 1994). We initiated a survey program of extended dust shells around optically bright carbon stars in the HIRAS images (cf. Izumiura et al. 1995). Here we show the most spectacular results, on $\mathrm{U}$ Ant, in Figure 2. The $60 \mu \mathrm{m}$ image shows a considerably extended central component (FWHM 55") with an additional component which is nearly circularly distributed around the central component at about $3^{\prime}$ in radius. In the $100 \mu \mathrm{m}$ image the central component has a FWHM of about $90^{\prime \prime}$, which implies that it is somewhat resolved, and it shows a further slight extension in the lowest three contours. We have confirmed that these features are also discernible in the original IRAS survey scan data.

We analysed the brightness distribution obtained on the basis of a double detached dust-shell model (Izumiura et al. 1997). It is reasonable to assume that the inner dust shell is also detached considering the existence 

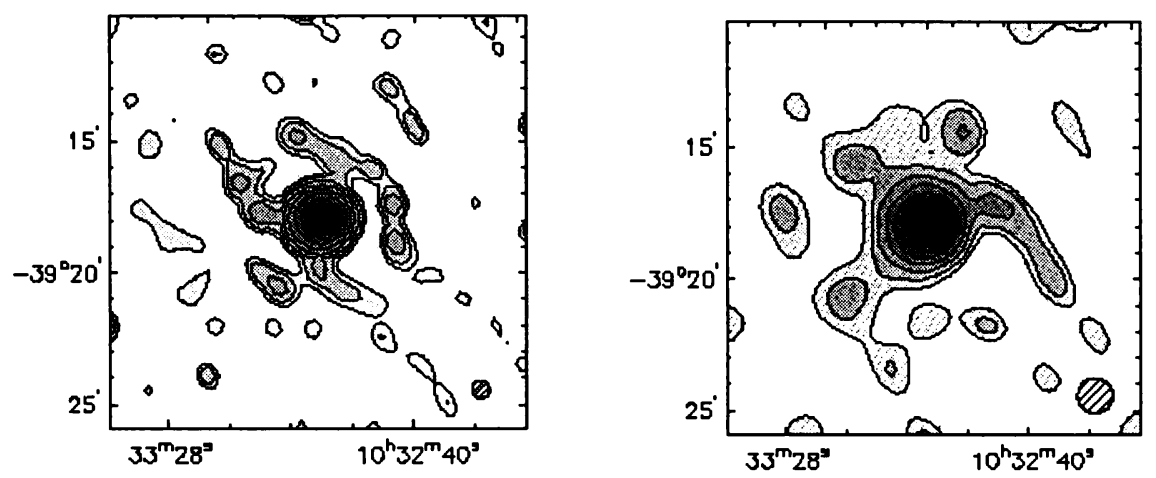

Figure 2. HIRAS images of U Ant in the $60 \mu \mathrm{m}$ band (left) and the $100 \mu \mathrm{m}$ band (right). Each image measures $16^{\prime} \times 16^{\prime}$. The contour levels are given in steps of factors of 2 in MJy sr ${ }^{-1}$ starting at $1 \mathrm{MJy} \mathrm{sr}^{-1}$. The hatched circle at the bottom-right corner shows the resolution (FWHM): $39^{\prime \prime}$ and $80^{\prime \prime}$ in the 60 and $100 \mu \mathrm{m}$ images, respectively.
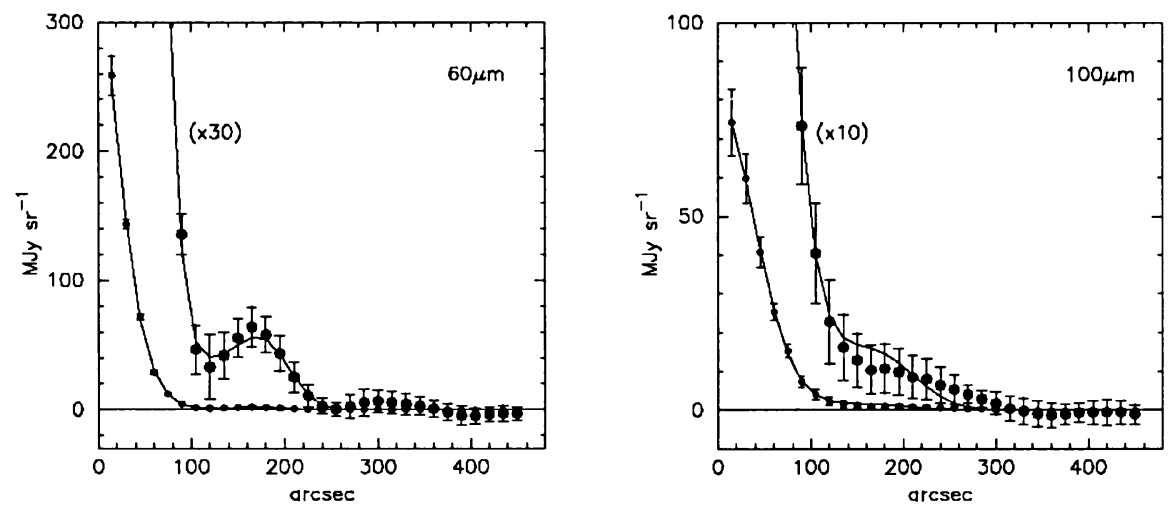

Figure 3. Simultaneous model fitting of a double dust shell to the brightness profiles of the dust shell of U Ant at $60 \mu \mathrm{m}$ (left) and $100 \mu \mathrm{m}$ (right). Filled circles are the data and the solid line shows the best fitting model for a shell thickness of $2.0 \times 10^{17} \mathrm{~cm}(3000$ years). The larger symbols show the same brightness profiles but thirty-and ten-times enlarged in the vertical direction in the left and right panels, respectively. Error bars show an estimated $1 \sigma$ uncertainty range.

of a detached $\mathrm{CO}$ gas envelope with a mean radius of $40^{\prime \prime}$ in this star (Olofsson et al. 1996). Simultaneous model fitting to the 60 and $100 \mu \mathrm{m}$ data is shown in Figure 3, which gives a shell separation of $6.0 \times 10^{17} \mathrm{~cm}$ and mass-loss rates in the inner and outer shells of $1.5 \times$ and $3.4 \times 10^{-6} \mathrm{M}_{\odot} \mathrm{yr}^{-1}$, respectively, for a thickness of $2.0 \times 10^{17} \mathrm{~cm}$ (i.e. a formation period of 3000 years with the expansion velocity of $21 \mathrm{~km} \mathrm{~s}^{-1}$ ) for both shells and a distance of $280 \mathrm{pc}$. The fitting results suggest that this star experienced two cycles of a periodic mass-loss variation on a time-scale of some $10^{4}$ years, the 

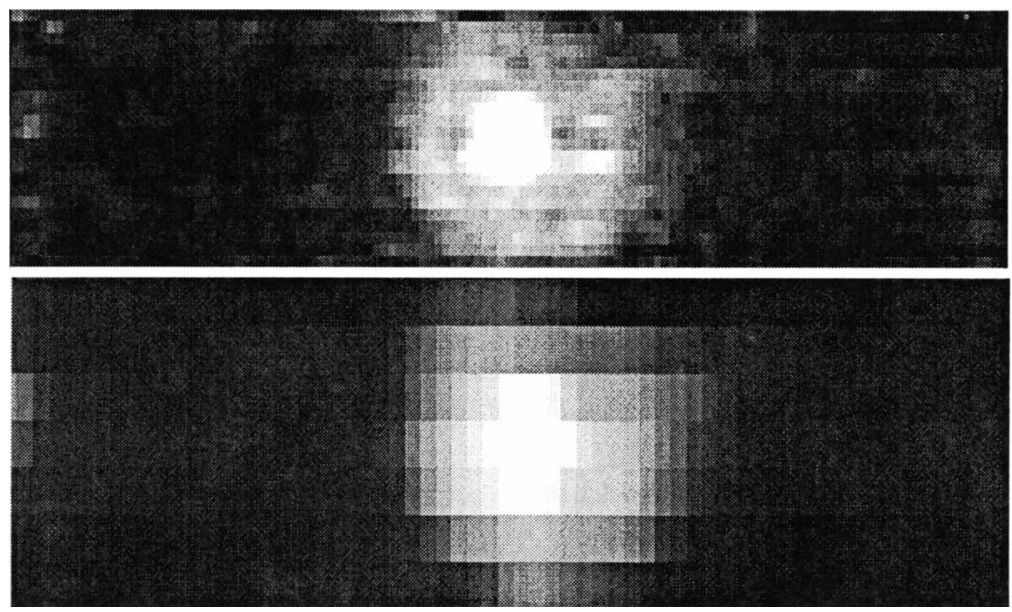

Figure 4. ISOPHOT images of Y CVn in the $90 \mu \mathrm{m}$ band (top) and $160 \mu \mathrm{m}$ band $\left(\right.$ bottom), where the pixel sizes are $15^{\prime \prime} \times 23^{\prime \prime}$ and $30^{\prime \prime} \times 92^{\prime \prime}$, respectively. The images measure $8^{\prime} .4 \times 32^{\prime} .0$ and $10^{\prime} .7 \times 32^{\prime} .0$
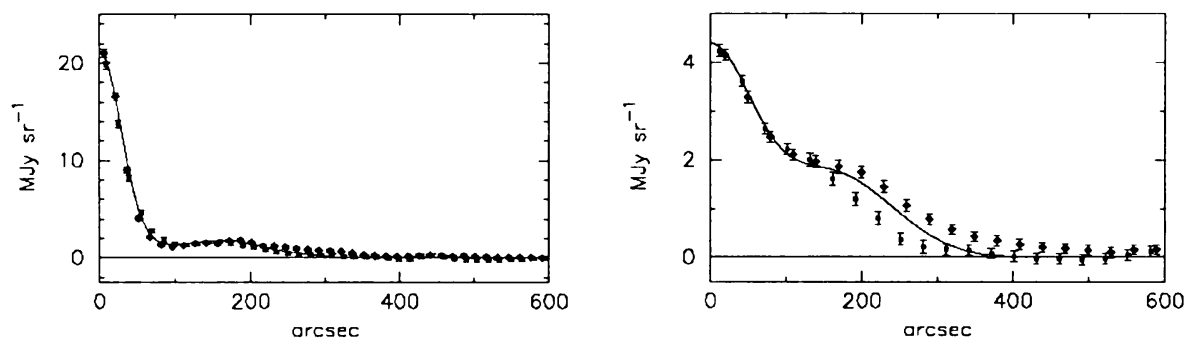

Figure 5. Brightness profiles of Y CVn at $90 \mu \mathrm{m}$ (left) and $160 \mu \mathrm{m}$ (right). Data points in the left half of the images are shown by squares and in the right half by diamonds. Error bars show only the statistical uncertainty. The solid line is a simultaneous model fit of a detached dust shell to the profiles in the two bands.

amplitude of which reached two orders of magnitude. Furthermore, relating the two shells directly to two consecutive thermal pulses allows us for the first time to determine the distance, interpulse period, core mass, and luminosity of the star self-consistently: they are $436 \mathrm{pc}, 1.4 \times 10^{4}$ years, $0.77 \mathrm{M}_{\odot}$, and $1.7 \times 10^{4} \mathrm{~L}_{\odot}$, respectively (Paczyński 1975; Izumiura et al. 1997).

\section{First ISO Images of the Dust Shell of Y CVn}

We are also mapping the dust shells of selected AGB stars in the far-infrared using the ISOPHOT imaging photo-polarimeter (Lemke et al. 1996) on 
board the Infrared Space Observatory. Y CVn was observed with the C100 camera through the C90 filter $\left(\lambda_{c}=95.1 \mu \mathrm{m}\right)$ and with the C200 camera through the $\mathrm{C} 160$ filter $\left(\lambda_{c}=174 \mu \mathrm{m}\right)$ using the AOT 32 oversampling mapping mode on 25 April 1996. The maps obtained are shown in Figure 4. In the $90 \mu \mathrm{m}$ map, sampling frequencies are $15^{\prime \prime}$ and $23^{\prime \prime}$ in the horizontal and vertical directions, respectively. In the $160 \mu \mathrm{m}$ map they are $30^{\prime \prime}$ and $92^{\prime \prime}$. The maps demonstrate the first direct evidence of a hollow dust shell surrounding an AGB star (Izumiura et al. 1996).

We have made a simultaneous model fit of a detached dust shell to the brightness profiles shown in Figure 5. It is found definitely necessary to introduce a hollow dust shell to reproduce the local brightness minimum observed in the $90 \mu \mathrm{m}$ data. The fit gives the mass-loss rate in the shell to be about $\sim 10^{-5} \mathrm{M}_{\odot} \mathrm{yr}^{-1}$, which is two orders of magnitude higher than the present-day mass-loss rate. The fitting results indicate that $\mathrm{Y}$ CVn underwent a sudden decline in mass-loss activity by two orders of magnitude $1.4 \times 10^{4}$ years ago (assuming a distace of $250 \mathrm{pc}$ ) and has been staying in that state.

With this picture of the mass-loss behavior, we conclude that the J-type carbon star Y CVn should be on the asymptotic giant branch. Our results will be important for understanding the evolutionary status of J-type carbon stars in the Galaxy.

The authors are indebted to the Digitized Sky Surveys that were produced at the Space Telescope Science Institute under U.S. Government grant NAG W-2166. The images of these surveys are based on photographic data obtained using the Oschin Schmidt Telescope on Palomar Mountain and the UK Schmidt Telescope.

\section{References}

Bontekoe, Tj. R., Koper, E. \& Kester, D. J. M. 1994, $A \& A p, 284,1037$

Gilman, R. C. $1972, A p J, 178,423$

Goldreich, P. \& Scoville, N. 1976, ApJ, 205, 144

Huggins, P. J., Olofsson, H. \& Johansson, L. E. B. 1988, ApJ, 332, 1009

Iben, I., Jr. \& Renzini, A. 1983, Ann. Rev. Astron. Astrophys., 21, 271

Izumiura, H., Hashimoto, O., Kawara, K., Yamamura, I. \& Waters, L. B. F. M. 1996, $A \& A p, 315, \mathrm{~L} 221$

Izumiura, H., Kester, D. J. M., de Jong, T., Loup, C., Waters, L. B. F. M. \& Bontekoe, Tj. R. 1995, ApESS, 224, 495

Izumiura, H., Waters, L.B.F.M., de Jong, T., Loup, C., Bontekoe, Tj.R. \& Kester, D. J. M. 1997, $A \& A p, 323,449$

Kwan, J. \& Hill, F. 1977, $A p J, 215,781$

Lemke, D., Klaas, U., Abolins, J., et al. 1996, $A \& A p$, 315, L64

Olofsson, H., Bergman, P., Eriksson, K. \& Gustafsson, B. 1996, $A \& A p, 311,587$

Paczyński, B. 1975, ApJ, 202, 558

Sopka, R. J., Hildebrand, R., Jaffe, D. T., Gatley, I., Roellig, T., Werner, M., Jura, M. \& Zuckerman, B. 1985, ApJ, 294, 242 


\section{Discussion}

Frogel: For U Ant, why should the shell flashes have produced dust shells? You need something special about the two flashes you claim have produced dust shells.

Izv niura: Each shell flash may have produced a dust shell. Or each shell fiash might have interrupted a high mass-loss phase. Neither can be ruled out at present. We need much higher spatial resolution to distinguish the two possibilities.

Little-Marenin: Y CVn has no Tc and hence is not likely to be an AGB star. Why do you think the two dust shells are produced by thermal pulses?

Izumiura: On the basis of the mass-loss behavior of Y CVn, it is almost indistinguishable from other AGB carbon stars. RGB stars usually show much lower mass-loss rates. Therefore we think the star is likely to be on the AGB in spite of the absence of Tc absorption lines. The star showing a double shell is not Y CVn but U Ant. U Ant has never been examined for Tc absorption lines. 


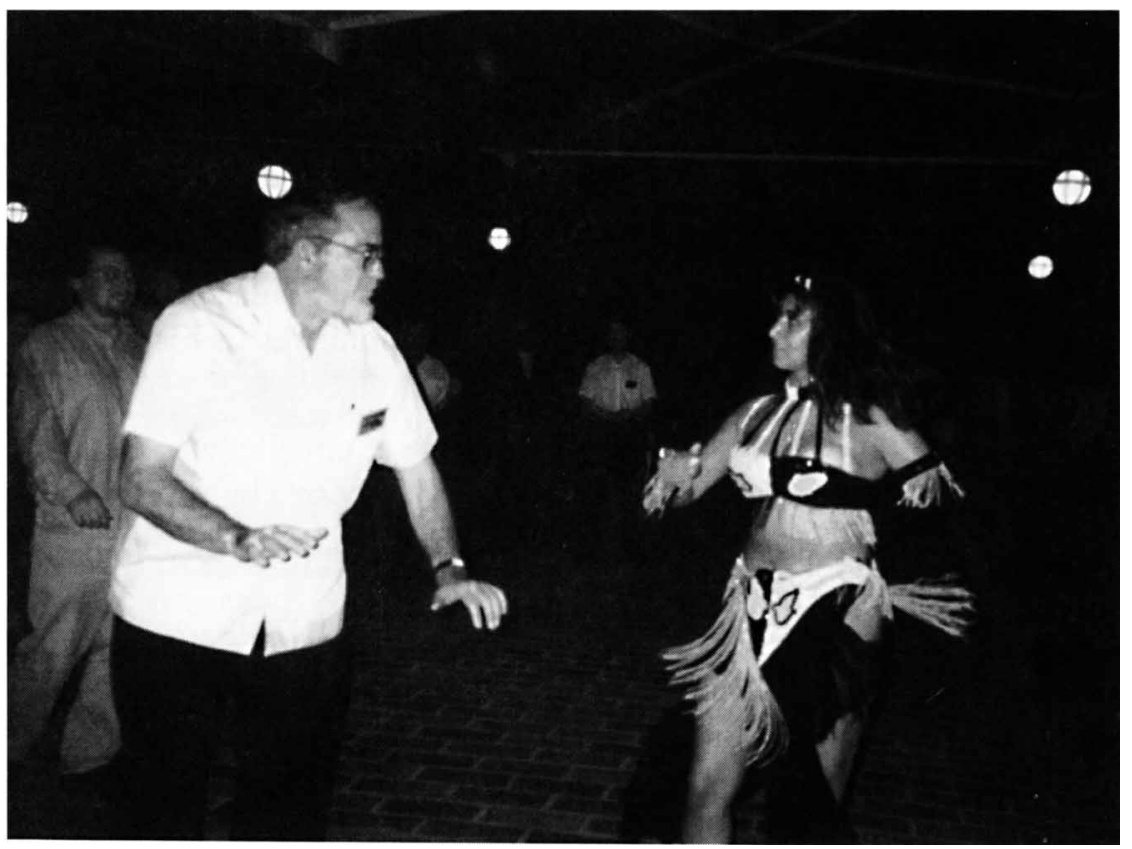

Instructions in belly-dancing were provided after the banquet. Here, SOC Chairman Robert Wing checks out the instructor. 